\title{
Visualization and quantification of nebulized aerosol deposition in mouth-lung casts under healthy and abnormal breathing conditions
}

\author{
Benjamin Wells ${ }^{1}$, Nathan Guest ${ }^{1}$, Jonathan Martinez ${ }^{1}$,Emmanuel Hakizimana ${ }^{1}$, Xiuhua April Si ${ }^{1}$, Wei-Chung Su ${ }^{2}$ and Jinxiang Xi $\mathrm{X}^{1,3 *}$ \\ ${ }^{1}$ Department of Aerospace and Mechanical Engineering, California Baptist University, Riverside, CA, 92504, USA \\ ${ }^{2}$ Department of Epidemiology, Human Genetics, \& Environmental Sciences, the University of Texas Health Science at Houston, Houston, TX 77225, USA \\ ${ }^{3}$ Department of Biomedical Engineering, California Baptist University, Riverside, CA, 92504, USA
}

\begin{abstract}
An accurate knowledge of drug distribution in human respiratory tract after drug administration is critical in establishing dose-response correlations and optimizing the treatment outcomes. However, human airways are inaccessible to conventional instruments, making it challenging to visualize and quantify regional or local depositions within. The objective of this study is to determine where nebulized drugs deposit in human upper airways, as well as how breathing conditions affect the outcome of pulmonary drug delivery. Sar-Gel was used to visualize the deposition pattern, and an electronic scale was used to quantify the deposited mass. Transient breathing conditions representative of the normal condition, asthma, and pulmonary fibrosis were considered separately using a breathing simulator. Computational modeling and simulations were also employed to cross-validate corresponding steady tests. An excellent match was obtained in the surface deposition between in vitro tests and numerical simulations. Sar-Gel deposition patterns vividly show deposition variation in response to different breathing conditions. A significant drug deposition was observed in the throat under normal breathing conditions. For obstructive lung diseases such as asthma (by increasing flow resistance), the data show a significant reduction of aerosol deposition in the upper airways. In contrast, the restrictive lung diseases such as pulmonary fibrosis (by decreasing lung compliance) gave rise to the similar amount deposited aerosol as the normal case, in spite of significantly different local deposition patterns between these two cases. Results of this study suggest that the Sar-Gel visualization approach in sectional respiratory tract casts effectively characterizes local deposition of nebulized droplets and can be used for future test of nebulizer performance.
\end{abstract}

\section{Introduction}

A key factor in respiratory drug delivery is to know where the medications end up and how many agents get to the target. Both the total and the local depositions are critical to the clinical outcome of inhalation therapies. Therefore, it is crucial to quantify the aerosol deposition distribution in different airway regions. However, the human respiratory tract is inaccessible to conventional visualization and quantification tools except for the radioactive imaging techniques. Such imaging techniques include gamma scintigraphy, Positron Emission Tomography (PET), and Single Photon Emission Computerized Tomography (SPECT). Two-dimensional or threedimensional aerosol distributions can be obtained using these imaging techniques, which have proven invaluable in testing the performance of inhalation devices [1]. However, one major setback of the imaging techniques is the radioactive risk exposed to the subjects [2,3]. Other setbacks include cost, availability, and operational complexities [2,3]. Numerical modeling and simulations have also been extensively used in the inhalation device design and environmental health risk assessment $[4,5]$. Flow details can be easily captured using numerical simulations, which are often difficult to obtain in experiments. However, inhalation drug delivery is a process that can be complicated by a variety of factors, such as inhalation devices, breathing conditions, patient health status, and drug formulations. Many assumptions are needed to make the modeling practical. In this sense, many compounding factors are neglected, which may or may not significantly alter the aerosol behaviors. It is also noted that current in vitro tests in quantifying dosimetry often utilized casts that are not transparent, nor can be opened apart. Therefore, visualization of the deposition distributions inside the casts is not feasible. It is important to develop a technique that can visualize and quantify the aerosol deposition within human respiratory tract in a simple, reliable, and safe manner [6].

Dalby and collaborators [7-9] have used Sar-Gel (Sartomer, PA) to visualize qualitatively aerosol deposition distributions in transparent airway casts. Sar-Gel is a chemical that changes into pink in contact with moisture and has been shown to be sensitive enough to a moisture mass as low as $0.5 \mu \mathrm{L}$ [7,9]. In several recent studies, $\mathrm{Xi}$ et al. $[3,10,11]$ used Sar-Gel to visualize the deposition distributions in sectional airway cast replicas using various types of spray pumps and nebulizers. A Sar-Gel deposition image shows different color levels depending on the deposited mass of the inhaled droplets. This colorimetry-mass correlation can be exploited to quantify the deposition and be used as an auxiliary method to the more complex and costly imaging approaches.

${ }^{*}$ Correspondence to: Jinxiang Xi, Department of Biomedical Engineering, Gordon and Jill Bourns College of Engineering, California Baptist University, 432 Magnolia Ave, Riverside, CA 92504, USA, Tel: (951) 552-8084; Fax: (951) 343-4972; E-mail: jxi@calbaptist.edu

Key words: pulmonary drug delivery, mouth-lung model cast, Sar-Gel visualization, aerosol deposition distribution, jet nebulize

Received: June 12, 2018; Accepted: June 21, 2018; Published: June 26, 2018 
Previous research has focused on the effectiveness of various drug administration tools such as different nebulizer types [12-15]. Excellent reviews of nebulizer usage and selection for patients with chronic obstructive pulmonary disease (COPD), asthma, and cystic fibrosis can be found in [16], [17], and [18], respectively. Even though such devices were shown to be effective for subjects who have minimal to no respiratory diseases, it is not clear how well they can administer drugs to patients with lung diseases such as asthma, COPD, acute respiratory distress syndrome (ARDS), or pulmonary fibrosis. Furthermore, most studies used a steady-state inhalation condition, which may not faithfully mimic the tidal breathing condition with unique flow features such as flow acceleration, deceleration, and reversal. The objective of this study is to visualize the deposition distribution of nebulized aerosols under physiologically realistic tidal breathing conditions, as well as to investigate the effects of pathological breathing conditions on aerosol deposition in human upper airways.

\section{Methods and materials}

\section{In vitro experiment design}

Software Magics (Materialise, Ann Arbor, MI) was used to prepare the hollow airway replicas from two mouth-lung model geometries that had been developed in our previous studies [3,11], as shown in Figure 1a. These hollow casts have a constant wall thickness of $4 \mathrm{~mm}$ [19]. The casts were divided into different sections to measure subregional deposition rates. Grooves were designed at the connecting ends to facilitate sealing and assembly between different parts [20,21]. The hollow casts were manufactured with a Stratasys Objet30 Pro 3-D printer (Northville, MI). This printer has a high printing resolution of $16 \mu \mathrm{m}$. Polypropylene, a transparent material, was utilized to fabricate the hollow model that the deposition patterns can be viewed from the outside of the casts. The $3 \mathrm{D}$ printed model was placed inside of a sealed chamber that was roughly the size of a lung.

Figure $1 \mathrm{~b}$ shows the experimental setup used for pulmonary drug delivery. The lung model was positioned in a 5-liter container that represents the typical adult lung volume [22]. A vacuum pump (Robinair, Warren, MI) and a breathing machine (Michigan Instruments, Grand Rapids, MI) were used to ventilate the container to simulate either steady or tidal breathing. The breath simulator can simulate the inhalation and exhalation processes that represented normal, asthma (obstructive lung disease), and pulmonary fibrosis (restrictive lung disease). The exhalation flow rate was measured using TSI 9565 VelociCalc ventilation meter (Shoreview, MN). Each test was repeated five times for later statistical analysis.

Before each test, the mouth-lung models were prepared by ensuring all parts were dry and then cataloged the weights for each part using an electronic balance (Cole-Parmer Symmetry PA-224E). The model was then sealed along the seams using $3 \mathrm{M}$ Transpore surgical tape which allowed for quick application and removal of the tape after the test but didn't leave a measurable residue that could corrupt the data. Aerosols from jet nebulizer (Philips Respironics InnoSpire Essence) were released into the mouth during the inhalation for 30 respiratory cycles. After the test is complete, the tape was quickly removed from the model and the components of the mouth-lung model were individually weighed before being cleaned. Deposited aerosol mass was calculated as the mass difference of the cast before and after the test.

To visualize aerosol deposition distributions, a thin layer of SarGel was applied on the inner wall of the model. Sar-Gel is a moisture indication paste that turns into pink upon contact with water. To ensure an evenly distributed coating, soft bristle brushes were used to eliminate Sar-gel ridges, which could trap moisture in an unrealistic manner. An electronic balance was used to measure the output per dosing as the mass difference before and after the spray release.

To simulate pathological breathing conditions, airflow resistor (PneuFlo Parabolic) and lung compliance were varied in the breath simulator. In order to simulate a patient with asthma the air resistors into and out of the lung were switched from the default Rp 5 (i.e., 5 $\mathrm{cm} \mathrm{H}_{2} \mathrm{O} / \mathrm{L} / \mathrm{s}$ for a healthy patient) to $\mathrm{Rp} 25$ (i.e., $25 \mathrm{~cm} \mathrm{H}_{2} \mathrm{O} / \mathrm{L} / \mathrm{s}$ for a patient with asthmatic symptoms), as shown in Figure 2. This led to a longer period of time to allow the lung to fully exhale before another breath was started (i.e., obstructive respiratory disease, with large slow breaths). To simulate a restrictive lung disease such as pulmonary fibrosis, the flow resistors were switched back to the Rp 5 and the lung compliance was adjusted from the control of $0.1 \mathrm{~L} / \mathrm{cm} \mathrm{H}_{2} \mathrm{O}$ (a health range being anywhere from 0.05 to $0.1 \mathrm{~L} / \mathrm{cm} \mathrm{H}_{2} \mathrm{O}$ ) to $0.03 \mathrm{~L} / \mathrm{cm} \mathrm{H}_{2} \mathrm{O}$ to approximate the increased stiffness in the lung parenchyma [23-25].

\section{(a) Pulmonary delivery and mouth-lung models}
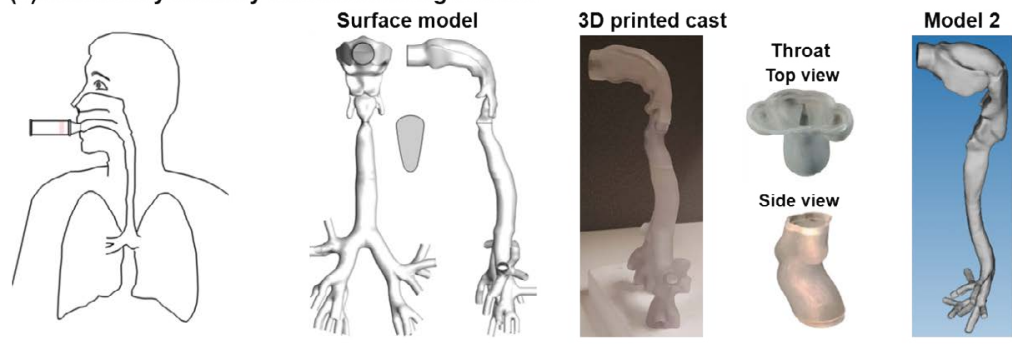

(b) Experimental setup
Steady inhalation
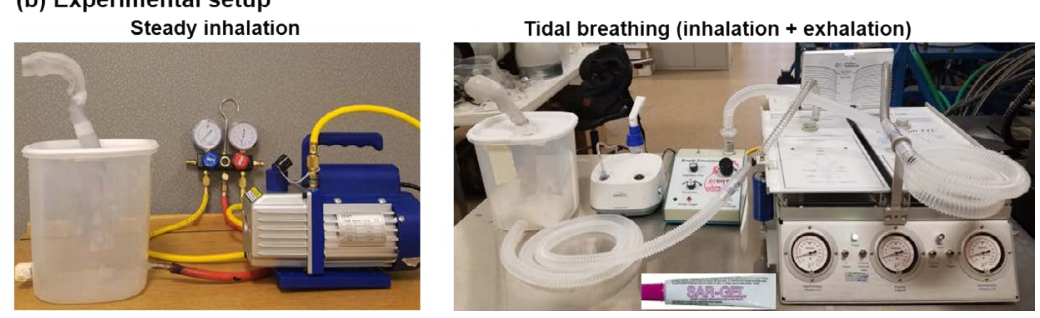

Figure 1. Experimental setup: (a) diagram of pulmonary drug delivery and mouth-lung models, (b) two ventilation modes: steady inhalation vs. tidal breathing with both inhalation and exhalation 


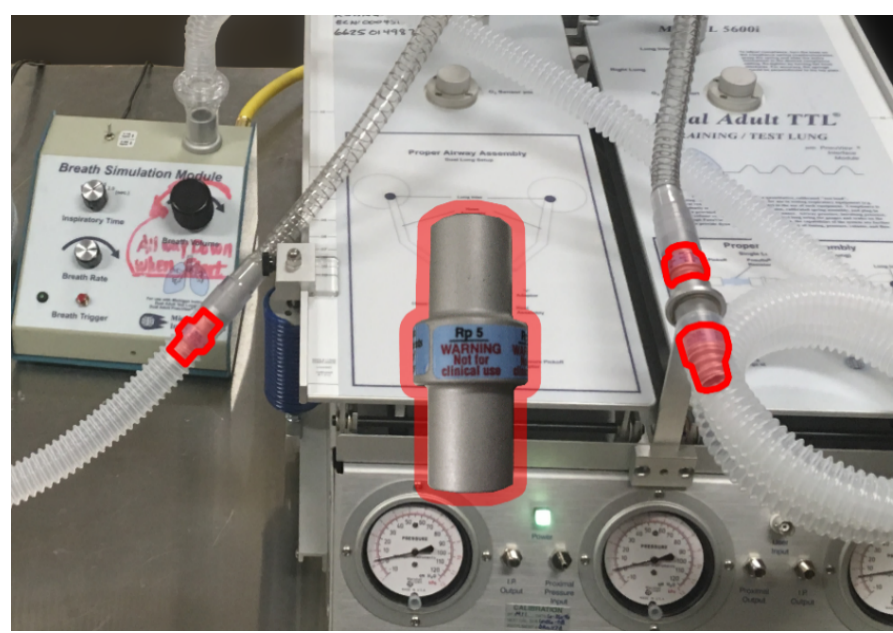

Figure 2. PneuFlo Parabolic flow resistor and the three locations they were used

\section{Computational simulations}

Complimentary computational simulations were performed in pulmonary drug delivery. Computational meshes were created using ANSYS ICEM 10 (Ansys, Inc.) with body-fitted elements near the inner airway surface. Grid independence study was conducted and the final computational mesh had 3.6 million elements [26]. The airflows were isothermal and incompressible, with flow regimes possible for laminar, transitional, and turbulent flows. To resolve this multi-regime flows, large eddy simulation (LES) was selected in this study due to its capacity to capture flow transitions and vortex details $[27,28]$. Chemical species transport model enhanced with finite particle inertia was employed to simulate the vapor behavior and fate [29,30]. Flow simulation package ANSYS Fluent (Canonsburg, PA) was used to simulate both flow and vapor dynamics.

\section{Statistical analysis}

Statistical analysis of deposition data was performed using software Minitab 17 (State College, PA). The data variability was assessed using One-Way Analysis of Variance (ANOVA) and Tukey's method with stacked data. Deposition results were presented as mean \pm standard deviations. If $\mathrm{p}$-value was $<0.05$ a difference was statistically significant.

\section{Results}

\section{Model validation: steady inhalation}

Comparison of deposition distributions between experimental tests and computational simulations is displayed in Figure 3. The inhalation flow rate was $20 \mathrm{~L} / \mathrm{min}$ and the nebulizer used was vibrating mesh type. From Figure $3 a$, an excellent match was obtained between the Sar-Gel generated deposition image and the computationally predicted deposition patterns, indicating that the computational modeling herein indeed captured the aerosol transport and deposition mechanisms. Particularly, there exist several positions with highly similar in vitro -CFD deposition patterns. First, CFD successfully predicted the crescent-shaped deposition hot spot in the subglottal region. The two streaks in the middle front trachea also matched closely between the experimental and computational results.

To quantify the sub-regional deposition, five sections are delineated: oral cavity, throat, trachea, G1, G2-3, and G4-5, as displayed in Figure $3 \mathrm{~b}$. The lumen volumes and surface areas of each region are listed in Figure $3 \mathrm{c}$. The area-to-volume ratio $\left(\mathrm{A}_{\mathrm{s}} / \mathrm{Vol}\right)$ denotes the complexity of the airway morphology. For instance, the area-to-volume ratio is 2.0 for G1, 2.7 for G1-2, and 4.6 for G4-5. The deposition fraction (DF) in each region is listed in the third column of Figure $3 \mathrm{~d}$. There are $7.1 \%$ depositing in G1 and 7.4\% in G2-3, in comparison to $25.7 \%$ in the oral cavity, $5.5 \%$ in the throat, and $9.1 \%$ in the trachea. The dose in each region per unit area was also computed and listed in the fourth column. The throat received the highest dose per unit area due to its flowlimiting nature. Downstream of the throat, the dose per area gradually decreases along the respiratory tract. This decrease results from two factors: (1) the depletion of inhaled vapor due to upstream deposition, and (2) the increased surface area and morphology from the trachea to G5.

Airflow field and vapor transport in the human upper airway for an inhalation flow rate of $20 \mathrm{~L} / \mathrm{min}$ are shown in Figure 4 . Considering the midplane view of the MT and TB regions (Figure 3a), the maximum speed is observed in the glottis, which has the minimum cross-sectional area and is the flow-limiting area in the human upper airway. Because of the jet effect of the laryngeal jet, recirculation zone forms in the dorsal region of the trachea (Figure $4 \mathrm{a}$ ). To demonstrate the effects of recirculation motion on the flow field, streamlines are plotted in the TB dorsal regions. These streamlines are highly heterogeneous because of the complex vortex patterns. Considering the vapor transport in Figure $3 \mathrm{~b}$ the vapor concentration is the highest at the inlet and decreases progressively due to the wall deposition. This decrease is faster at lower inhalation flow rates because of the prolonged vapor residence time but is significantly minimized at high flow rates (say $40 \mathrm{~L} / \mathrm{min}$ ).
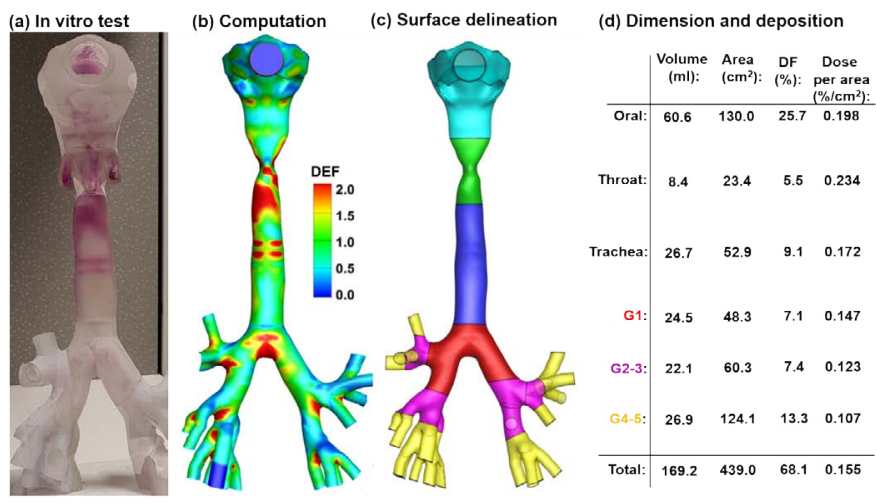

Figure 3. Comparison of deposition distributions in the mouth-lung geometry between experiments and computational predictions: (a) in vitro tests, (b) computational prediction, (c) surface divided into different sections, and (d) dimension and deposition of different sections
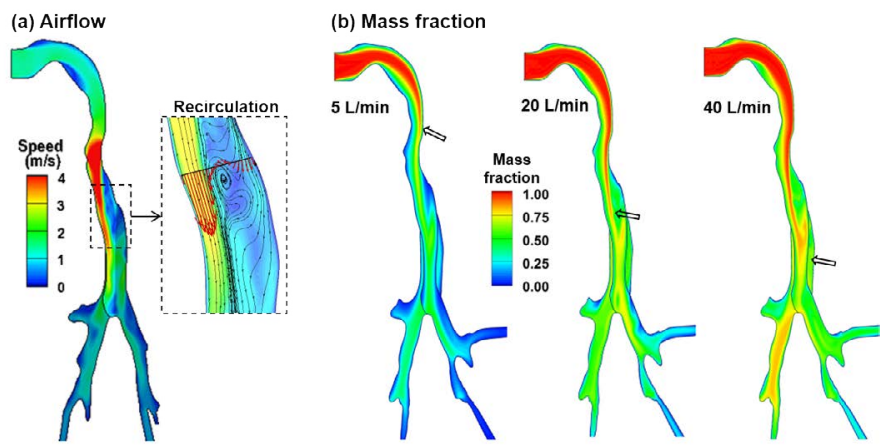

Figure 4. Airflow and vapor transport in the mouth-lung model: (a) airflow field and (b) mass fraction of the inhaled vapor species at different inhalation rates: $5 \mathrm{~L} / \mathrm{min}, 20 \mathrm{~L} / \mathrm{min}$, $40 \mathrm{~L} / \mathrm{min}$ 


\section{Tidal breathing}

Figure 5a shows the bod plots of the in vitro deposition results in the model 2 under normal and pathological breathing conditions. The aerosol administration duration was 30 respiration cycles. It was observed that the dose in the asthma case was significantly lower than ( $~ 50 \%$ of $)$ that in the normal case. In contrast, the fibrosis case gave rise to a similar upper airway dose as the normal case. Considering the data variability, even though the deposition data exhibited a relatively large range of fluctuation (especially for the normal case), no outlier was observed for the three cases considered, indicating satisfactory repeatability of the deposition data.

To better understand the above deposition variation, the expiration flow velocities were also measured for each test, as shown in Figure 5b. as expected, a much lower expiratory flow rate was observed in the asthma case, where the elevated resistance remarkably deterred the gas movement and thus prolonged the exhalation process. A similarly low expiration flow rate was also noted in the fibrosis case, presumably due to a reduced tidal volume per se. During the inhalation, the decreased lung compliance (i.e., a stiffer lung) would restrict the airflow, making the breath small and brief.

Surface deposition patterns of nebulized aerosols are compared in Figure 6 among the three breathing conditions considered. As with Figure 5, a much darker color was observed in the normal case, which was followed by the fibrosis case, while the asthma case had the least dark color. Compared to the normal case, the shallow breath in fibrosis led to a decreased deposition in the lungs, as illustrated in the right lower region between Figure $6 \mathrm{a}$ and Figure $6 \mathrm{~b}$. Interestingly, the left and right surface depositions were not symmetric for all cases considered.

Sub-regional doses in the four parts of the airway are shown in Figure 7. It was evident that deposition distribution was very sensitive to the ventilation (i.e., different flow resistance and lung compliance). The left-right asymmetry in deposited doses should be a result of asymmetric ventilations to the left and right lungs. Again, the data variability herein was within the satisfactory range with no apparent data outlier, which suggested good repeatability in light of the in vitro measurements.

\section{Discussion and conclusions}

Highly heterogeneous deposition profiles of inhaled vapor were observed for all inhaler devices and breathing conditions considered. Excellent agreements were achieved between the Sar-Gel generated surface deposition image and the computationally predicted vapor deposition pattern in the lung casts, instilling confidence into this newly proposed visualization method. Notably, both the experiment and simulation captured the crescent-shaped deposition hot spots downstream of the throat and the two streaks in the middle trachea (Figure 3a). The proposed Sar-Gel approach can be readily modified
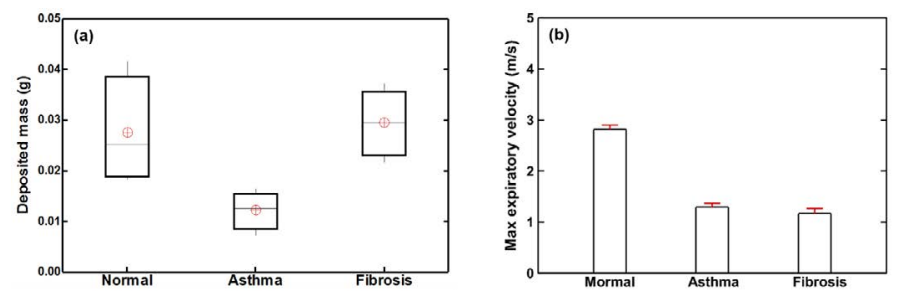

Figure 5. In vitro tests with tidal ventilation for both normal and pathological conditions in mouth-lung model 2: (a) box plot of the deposited mass for 30 respiration cycles and (b) maximum expiratory airflow velocity at the mouth opening (a) Normal

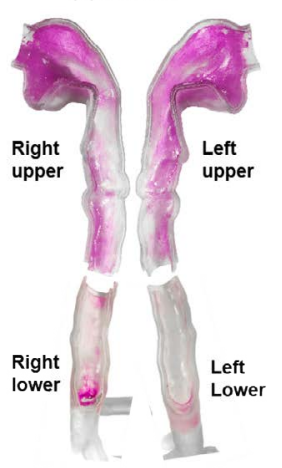

(b) Asthma

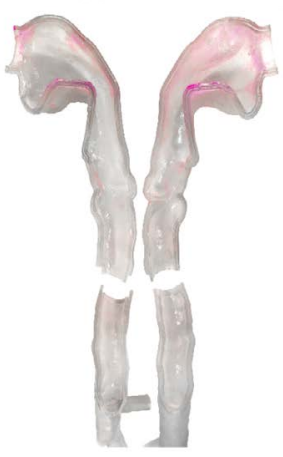

(c) Fibrosis

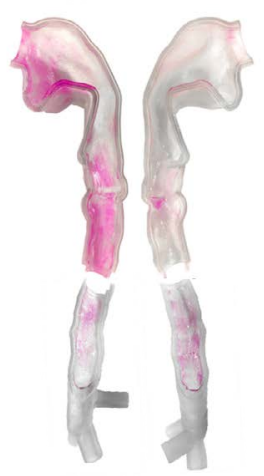

Figure 6. Comparison of surface deposition distribution of nebulized aerosols under different breathing conditions representative of (a) normal, (b) asthma (obstructive lung disease with elevated resistance), and (3) pulmonary fibrosis (restrictive lung disease with low compliance)

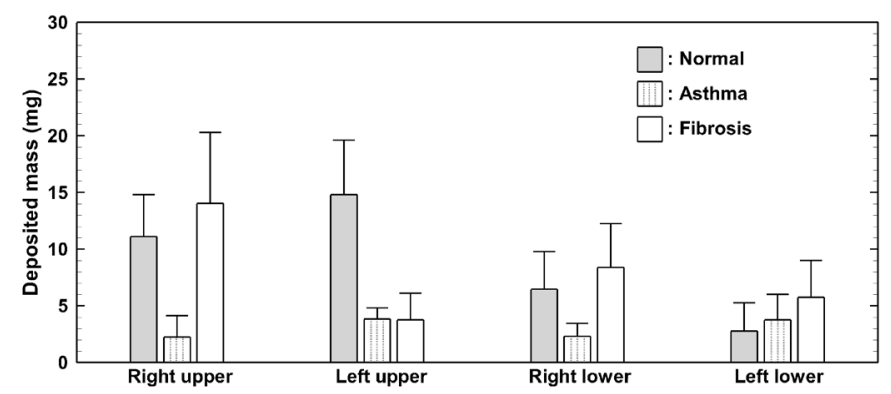

Figure 7. Deposited mass of nebulized aerosol in sub-regions for 30 reparation cycles. The delineation of the four sub-regions is shown in Figure 6

to investigate drug dispensing to other regions in the respiratory tract, using different devices, and with different delivery protocols.

Taking the data together, two factors appear to have major effects on the deposition of nebulized aerosols. Firstly, the geometrical realism and anatomical details are crucial to the aerosol deposition. The complex nature of the upper respiratory tract suggests that a majority of the aerosol will undesirably deposit on the walls with shape geometry changes. Second, the health condition of the patient is important. Compared to the normal breathing condition, the asthma model had far less deposited mass. This can be related to the much lower airflow velocity for the asthma case (Figure $5 b$ ), which is in turn caused by the higher flow resistance. By contrast, the pulmonary fibrosis model (restrictive lung disease) gave rise to a similar amount of deposited aerosol as the normal case.

To reliably quantify the total and olfactory deposition rates, it is desirable to use a shell-like airway cast as implemented in this study (Figure 1) as opposed to the conventional block-type casts. In most previous studies, airway cast replicas were developed by subtracting a block with the airway to obtain the airway space within the solid block $[20,21,31]$. The weights of such block-type casts are typically close to or beyond the capacity of high-precision lab scales. For instance, the maximum weight capacity of the scale used in this study (Sartorius $\mathrm{R} 160 \mathrm{P}, 0.01 \mathrm{mg}$ precision) is $160 \mathrm{~g}$. A low precision (1mg and above) scale cannot reliably measure the differential weight before and after the nebulizer administration due to the low dosages. This requirement is more pronounced when measuring the regional deposition rate. Another issue is that the measurement of such small quantities can be affected by the slightest variations in the surrounding, such as noise, 
vibration, breeze, magnetic field, temperature, and humidity. Care was taken in this study to minimize such influences. The measurements were conducted in a quiet room free from noise and vibration. After each test, the cast was washed, dried, and left in the lab for another 30 minutes to allow the cast became equilibrium with the environment. These procedures were noted to be important to eliminate complications from humidity and temperature; substantial fluctuations had been recorded otherwise due to vapor evaporation or absorption. However, such procedures would take about 45 minutes for each test if there were no other issues that arose, thereby making it highly time-consuming to collect sufficient measurements to be statistically significant.

Sar-Gel visualization method and numerical modeling can be complementary to each other in the optimization of inhalation protocols. The Sar-Gel shows the distribution of aerosol deposition in a direct and qualitative manner, whereas the computational simulations can provide details of the airflow and aerosol behaviors behind the observed aerosol deposition. While computational simulations are more cost-efficient, they are limited by many simplifications that inevitably reduced their physical realism to represent the in vivo or experimental scenarios. To this end, the Sar-Gel method can provide more clinically relevant results and include realistic factors that are still difficult, if not prohibitive, to computational methods. Although possible to be considered in CFD, these factors require either advanced algorithms or large resources. Recent efforts have been taken to numerically include the effects of polydisperse aerosols [32,33], electric charges [34-36], hygroscopic growth [37,38], compliant geometries $[36,39]$, more bronchiole generations $[40,41]$, glottal motion [42-44], and inter-subject uncertainties $[45,46]$.

In summary, deposition of nebulized droplets from a jet nebulizer in anemically accurate mouth-lung casts was characterized for both steady and transient ventilations and under both healthy and pathological conditions. Specific findings include:

1. Steady-state deposition distributions agreed well between in vitro tests and numerical simulations.

2. Sar-Gel deposition patterns exhibited significant heterogeneity in response to different breathing conditions

3. For asthma with elevated flow resistance, there was a substantial reduction in the aerosol deposition in the upper airways in comparison to the normal case.

4. For fibrosis with lower lung compliance, similar amount deposited aerosol was observed as the normal case, even though their deposition distributions are very different.

5. Sar-Gel visualization method can be used as a platform to design and test new devices or delivery protocols.

\section{References}

1. Dolovich MB (2001) Measuring total and regional lung deposition using inhaled radiotracers. J Aerosol Med 14 Suppl 1: S35-44. [Crossref]

2. Darquenne C, Fleming JS, Katz I, et al. (2016) Bridging the Gap Between Science and Clinical Efficacy: Physiology, Imaging, and Modeling of Aerosols in the Lung. $J$ Aerosol Med Pulm Drug Deliv 29: 107-12. [Crossref]

3. Xi J, Yuan JE, Zhang Y, Nevorski D, Wang Z, et al. (2016) Visualization and Quantification of Nasal and Olfactory Deposition in a Sectional Adult Nasal Airway Cast. Pharm Res 33: 1527-1541. [Crossref]

4. Zhang Z, Martonen TB (1997) Deposition of ultrafine aerosols in human tracheobronchial airways. Inhal Toxicol 9: 99-110.

5. Inthavong K, Tian ZF, Li HF, Tu JY, Yang W, et al. (2006) A numerical study of spray particle deposition in a human nasal cavity. Aerosol Sci Technol 40: 1034-U1033.
6. Inthavong K, Tao Y, Petersen P, Mohanarangam K, Yang W, et al. (2016) A smoke visualisation technique for wake flow from a moving human manikin. J Vis 19: 1-13.

7. Kundoor V, Dalby RN (2011) Effect of formulation- and administration-related variables on deposition pattern of nasal spray pumps evaluated using a nasal cast. Pharm Res 28:1895-1904. [Crossref]

8. Guo Y, Laube B, Dalby R (2005) The effect of formulation variables and breathing patterns on the site of nasal deposition in an anatomically correct model. Pharm Res 22: 1871-1878. [Crossref]

9. Kundoor V, Dalby RN (2010) Assessment of nasal spray deposition pattern in a silicone human nose model using a color-based method. Pharm Res 27: 30-36. [Crossref]

10. Xi J, Wang Z, Nevorski D, White T, Zhou Y (2017) Nasal and olfactory deposition with normal and bidirectional intranasal delivery techniques: in vitro tests and numerical simulations. J Aerosol Med Pulm Drug Deliv 30: 118-131. [Crossref]

11. Xi J, Yang T, Talaat K, Wen T, Zhang Y, et al. (2018) Visualization of local deposition of nebulized aerosols in a human upper respiratory tract model. J Vis 21: 225-237.

12. Butz AM, Tsoukleris MG, Donithan M, Hsu VD, Zuckerman I, et al. (2006) Effectiveness of nebulizer use-targeted asthma education on underserved children with asthma. Arch Pediatr Adolesc Med 160: 622-628. [Crossref]

13. Stein SW, Thiel CG2 (2017) The History of Therapeutic Aerosols: A Chronological Review. J Aerosol Med Pulm Drug Deliv 30: 20-41. [Crossref]

14. Shi S, Dodds Ashley ES, Alexander BD, Hickey AJ (2009) Initial characterization of micafungin pulmonary delivery via two different nebulizers and multivariate data analysis of aerosol mass distribution profiles. AAPS PharmSciTech 10: 129. [Crossref]

15. Sims MW (2011) Aerosol therapy for obstructive lung diseases: device selection and practice management issues. Chest 140: 781-788. [Crossref]

16. Tashkin DP (2016) A review of nebulized drug delivery in COPD. Int J Chron Obstruct Pulmon Dis 11: 2585-2596. [Crossref]

17. Lin YZ, Hsieh KH (1995) Metered dose inhaler and nebuliser in acute asthma. Arch Dis Child 72: 214-218. [Crossref]

18. Denyer J, Prince I, Dixon E, Agent P, Pryor J, et al. (2010) Evaluation of the Target Inhalation Mode (TIM) breathing maneuver in simulated nebulizer therapy in patients with cystic fibrosis. J Aerosol Med Pulm Drug Deliv 23 Suppl 1: S29-36. [Crossref]

19. Xi J, Berlinski A, Zhou Y, Greenberg B, Ou X (2012) Breathing resistance and ultrafine particle deposition in nasal-laryngeal airways of a newborn, an infant, a child, and an adult. Ann Biomed Eng 40: 2579-2595. [Crossref]

20. Zhou Y, Xi J, Simpson J, Irshad H, Cheng Y-S (2013) Aerosol deposition in a nasopharyngolaryngeal replica of a 5-year-old child. Aerosol Sci Technol 47: 275-282.

21. Zhou Y, Guo M, Xi J, Irshad H, Cheng YS (2014) Nasal deposition in infants and children. J Aerosol Med Pulm Drug Deliv 27: 110-116. [Crossref]

22. Jones RL, Nzekwu MM (2006) The effects of body mass index on lung volumes. Chest 130: 827-833. [Crossref]

23. Bernasconi M, Ploysongsang Y, Gottfried SB, Milic-Emili J, Rossi A(1988) Respiratory compliance and resistance in mechanically ventilated patients with acute respiratory failure. Intensive Care Med 14: 547-553. [Crossref]

24. Storstein O, Field AS Jr, Massumi R, Gray FD Jr (1959) Airway resistance and lung compliance; the effects of histamine and hyperventilation in normal subjects and patients with chronic lung disease. Yale J Biol Med 31: 387-396. [Crossref]

25. Papandrinopoulou D1, Tzouda V, Tsoukalas G (2012) Lung compliance and chronic obstructive pulmonary disease. Pulm Med 2012: 542769. [Crossref]

26. Xi J, Kim J, Si XA, Zhou Y (2013) Diagnosing obstructive respiratory diseases using exhaled aerosol fingerprints: A feasibility study. J Aerosol Sci 64: 24-36.

27. Xi J, Si X, Kim J, Su G, Dong H (2014) Modeling the pharyngeal anatomical effects on breathing resistance and aerodynamically generated sound. Med Biol Eng Comput 52: 567-577. [Crossref]

28. Si X, Xi J, Kim J (2013) Effect of laryngopharyngeal anatomy on expiratory airflow and submicrometer particle deposition in human extrathoracic airways. Open J Fluid D 3: 286-301.

29. Dhand R (2002) Nebulizers that use a vibrating mesh or plate with multiple apertures to generate aerosol. Respir Care 47: 1406-1416. [Crossref]

30. Waldrep JC, Dhand R (2008) Advanced nebulizer designs employing vibrating mesh/aperture plate technologies for aerosol generation. Curr Drug Deliv 5:114-119. [Crossref] 
Wells B (2018) Visualization and quantification of nebulized aerosol deposition in mouth-lung casts under healthy and abnormal breathing conditions

31. E Taoum KK, Xi J, Kim J, Berlinski A (2015) In Vitro Evaluation of Aerosols Delivered via the Nasal Route. Respir Care 60: 1015-1025. [Crossref]

32. Xi J, Yuan JE, Si XA (2016) Simulation study of electric-guided delivery of $0.4 \mu \mathrm{m}$ monodisperse and polydisperse aerosols to the ostiomeatal complex. Comput Biol Med 72: 1-12. [Crossref]

33. Rosati JA, Brown JS, Peters TM, Leith D, Kim CS (2002) A polydisperse aerosol inhalation system designed for human studies. J Aerosol Sci 33: 1433-1446.

34. Bailey AG (1997) The inhalation and deposition of charged particles within the human lung. J Electrostat 42: 25-32.

35. Xi J, Si X, Longest W (2014) Electrostatic charge effects on pharmaceutical aerosol deposition in human Nasal-Laryngeal airways. Pharmaceutics 6:26-35. [Crossref]

36. Xi J, Yuan J, Alshaiba M, Cheng D, Firlit Z, et al. (2016) Design and testing of electricguided delivery of charged carticles to the olfactory region: experimental and numerical studies. Curr Drug Deliv 13:265-274. [Crossref]

37. Zhang Z, Kleinstreuer C, Kim CS (2006) Water vapor transport and its effects on the deposition of hygroscopic droplets in a human upper airway model. Aerosol Sci Technol 40: 52-67.

38. Kim JW, Xi J, Si XA (2013) Dynamic growth and deposition of hygroscopic aerosols in the nasal airway of a 5-year-old child. Int J Numer Method Biomed Eng 29: 17-39. [Crossref]

39. Talaat K, Xi J (2017) Computational modeling of aerosol transport, dispersion, and deposition in rhythmically expanding and contracting terminal alveoli. $J$ Aerosol Sci 112:19-33.
40. Xi J, Zhao W, Yuan JE, Cao B, Zhao L (2017) Multi-resolution classification of exhaled aerosol images to detect obstructive lung diseases in small airways. Comput Biol Med 87:57-69. [Crossref]

41. Corley RA, Kabilan S, Kuprat AP, Carson JP, Minard KR, et al. (2012) Comparative computational modeling of airflows and vapor dosimetry in the respiratory tracts of rat, monkey, and human. Toxicol Sci 128: 500-516. [Crossref]

42. Xi J, April Si X, Dong H, Zhong H (2018) Effects of glottis motion on airflow and energy expenditure in a human upper airway model. Eur J Mech B Fluids 72: 23-37.

43. Scheinherr A, Bailly L, Boiron O, Lagier A, Legou T, et al. (2015) Realistic glottal motion and airflow rate during human breathing. Med Eng Phys 37: 829-839. [Crossref]

44. Xi J, Wang Z, Talaat K, Glide-Hurst C, Dong H (2018) Numerical study of dynamic glottis and tidal breathing on respiratory sounds in a human upper airway model. Sleep Breath 22: 463-479. [Crossref]

45. Walenga RL, Longest PW, Kaviratna A, Hindle M (2017) Aerosol drug delivery during noninvasive positive pressure ventilation: effects of intersubject variability and excipient enhanced growth. J Aerosol Med Pulm Drug Deliv 30: 190-205. [Crossref]

46. Lu J, Xi J, Langenderfer JE (2017) Sensitivity analysis and uncertainty quantification in pulmonary drug delivery of orally inhaled pharmaceuticals. J Pharm Sci 106: 3303 3315. [Crossref]

Copyright: (C2018 Wells B. This is an open-access article distributed under the terms of the Creative Commons Attribution License, which permits unrestricted use, distribution, and reproduction in any medium, provided the original author and source are credited. 